Article

\title{
Complex Symmetric Formulation of Maxwell Equations for Fields and Potentials
}

\author{
George Livadiotis
}

Southwest Research Institute, Space Science \& Engineering, San Antonio, TX 78238, USA; glivadiotis@swri.edu; Tel.: +1-210-274-4028

Received: 14 May 2018; Accepted: 19 June 2018; Published: 3 July 2018

check for updates

\begin{abstract}
Maxwell equations have two types of asymmetries between the electric and magnetic fields. The first asymmetry is the inhomogeneity induced by the absence of magnetic charge sources. The second asymmetry is due to parity. We show how both asymmetries are naturally resolved under an alternative formulation of Maxwell equations for fields or potentials that uses a compact complex vector operator representation. The developed complex symmetric operator formalism can be easily applied to performing the continuity equation, the field wave equations, the Maxwell equations for potentials, the gauge transformations, and the 4-momentum representation; in general, the developed formalism constitutes a simple way of unfolding the Maxwell theory. Finally, we provide insights for extending the presented analysis within the context of (i) bicomplex numbers and tessarine algebra; and (ii) $L^{p}$-spaces in nonlinear Maxwell equations.
\end{abstract}

Keywords: Maxwell equations; complex representation; E/M waves; gauge transformation; gravitomagnetism; $L^{p}$ norms

\section{Introduction}

There are various representations of Maxwell equations. Some examples are the following: standard complex representation [1,2], spinor form [3], Silberstein-Bateman-Majorana form [4-6], Kemmer-Duffin-Petiau form (also known as the meson algebra) [4,7], matrix representation [8], Dirac form [9-11], Poincaré algebra [12], Debye sources [13,14], Penrose's transformation presented in terms of integral geometry [15,16], integral representation [17], and multipolar presentation [18].

This paper uses the complex vector representation of Maxwell equations in order to develop the presented complex operator formalism. This developed formalism: (i) emerges naturally from the symmetry between electric and magnetic fields; and (ii) exhibits a compact set of equations for the fields and their potentials.

Most importantly, the presented formulation of Maxwell equations constitutes a much simpler and compact way of unfolding the Maxwell theory compared to previous complex formulations (e.g., continuity equation, wave equations, Maxwell equations for potentials, gauge symmetry).

The presented formulation of Maxwell equations constitutes a much simpler way of unfolding the Maxwell theory compared with previous complex formulations (e.g., continuity equation, wave equations, Maxwell equations for potentials, gauge symmetry). The analysis can trigger several theoretical developments and applications different from the standard Maxwell equations. Indeed, in the last section, we expose two examples where the presented analysis can be applied and extended, that is, within the context of (i) bicomplex numbers and tessarine algebra; and (ii) $L^{p}$-spaces in nonlinear Maxwell equations.

Next, in Section 2, we present the compact complex formalism of Maxwell equations. In Section 3, we apply this formalism in the derivations of the basic concepts of (i) continuity equation; (ii) wave equations; (iii) Maxwell equations for potentials; (iv) gauge transformation; and (v) 4-momentum 
of electromagnetic field. In Section 4, we summarize the conclusions, while in Section 5, we discuss what's next for further theoretical developments and applications of this formalism.

\section{Compact Complex Representation of Maxwell Equations}

The differential formalism of Maxwell equations for the electric $\vec{E}$ and magnetic $\vec{B}$ fields in the presence of electric charge sources with density $\rho_{e}$ and current $\overrightarrow{\mathbf{J}}_{\boldsymbol{e}}$, are written as:

$$
\begin{gathered}
\vec{\nabla} \cdot \vec{E}=\frac{\rho_{e}}{\varepsilon_{0}} \\
\mu_{0} \varepsilon_{0} \frac{\partial \vec{E}}{\partial t}=\vec{\nabla} \times \vec{B}-\mu_{0} \overrightarrow{\mathbf{J}}_{e} \\
\vec{\nabla} \cdot \vec{B}=0 \\
\frac{\partial \vec{B}}{\partial t}=-\vec{\nabla} \times \vec{E}
\end{gathered}
$$

which is consistent [19] with the sources continuity equation (by applying $\partial / \partial t$ and $-\vec{\nabla}$ to (1a) and (1b), respectively, then, summing):

$$
\frac{\partial \rho_{e}}{\partial t}+\vec{\nabla} \cdot \overrightarrow{\mathbf{J}}_{e}=0
$$

(Note: Electric/magnetic charge sources are denoted in bold letters throughout the letter.) The first two equations are inhomogeneous due to the electric charge sources. The source-free equations are homogeneous, but still suffer from the units and parity asymmetry:

$$
\begin{gathered}
\vec{\nabla} \cdot \vec{E}=0 \\
\mu_{0} \varepsilon_{0} \frac{\partial \vec{E}}{\partial t}=\vec{\nabla} \times \vec{B} \\
\vec{\nabla} \cdot \vec{B}=0 \\
\frac{\partial \vec{B}}{\partial t}=-\vec{\nabla} \times \vec{E}
\end{gathered}
$$

The units asymmetry can be easily resolved by setting the spatial coordinates $(c t, x, y, z)$ and the magnetic field $\vec{c} \vec{B}$. In this way, the:

$$
\begin{gathered}
\vec{\nabla} \cdot \vec{E}=0 \\
\frac{\partial \vec{E}}{\partial c t}=\vec{\nabla} \times c \vec{B} \\
\vec{\nabla} \cdot c \vec{B}=0 \\
\frac{\partial c \vec{B}}{\partial c t}=-\vec{\nabla} \times \vec{E}
\end{gathered}
$$

(where we used $c^{2} \mu_{0} \varepsilon_{0}=1$ ).

The parity transformation flips the sign of spatial coordinates, $\hat{P} \vec{r}=-\vec{r}$. The electric field has parity -1 (as any vector), while the magnetic field has parity +1 (as any axial vector, defined by a curl of a vector). Using the complex Riemann-Silberstein vector field $\vec{G} \equiv \vec{E}+i c \vec{B}$ [20] and Minkowski 
metric [21], the spacetime is represented by four equivalent spatial components (ict, $x, y, z)$ and the parity of $i c \vec{B}$ is -1 (as any regular vector),

$$
\begin{gathered}
\vec{\nabla} \cdot \vec{E}=0 \\
\frac{\partial \vec{E}}{\partial i c t}=-\vec{\nabla} \times i c \vec{B} \\
\vec{\nabla} \cdot i c \vec{B}=0 \\
\frac{\partial i c \vec{B}}{\partial i c t}=-\vec{\nabla} \times \vec{E}
\end{gathered}
$$

The Maxwell equations in (5) are symmetric. In fact, they can be compacted to:

$$
\begin{gathered}
\vec{\nabla} \cdot \vec{G}=0 \\
\left(\frac{\partial}{\partial i c t}+\vec{\nabla} \times\right) \vec{G}=0
\end{gathered}
$$

by setting:

$$
\vec{G} \equiv \vec{E}+i c \vec{B}
$$

(where $i c \vec{B}$ and $\vec{G}$ are vectors having parity -1 ).

The corresponding equations with electric charge sources are:

$$
\begin{gathered}
\vec{\nabla} \cdot \vec{E}=\frac{\rho_{e}}{\varepsilon_{0}} \\
\frac{\partial \vec{E}}{\partial i c t}=-\vec{\nabla} \times i c \vec{B}+\mu_{0} i c \overrightarrow{\mathbf{J}}_{e} \\
\vec{\nabla} \cdot i c \vec{B}=0 \\
\frac{\partial i c \vec{B}}{\partial i c t}=-\vec{\nabla} \times \vec{E}
\end{gathered}
$$

which can be compacted, analogously to Equation (6):

$$
\begin{gathered}
\vec{\nabla} \cdot \vec{G}=\frac{\rho_{e}}{\varepsilon_{0}} \\
\left(\frac{\partial}{\partial i c t}+\vec{\nabla} \times\right) \vec{G}=\mu_{0} i c \overrightarrow{\mathbf{J}}_{e}
\end{gathered}
$$

The Maxwell equations in Equation (8) are still asymmetric due to the absence of magnetic charge sources (monopoles). If there were monopoles, Maxwell equations would be written in the symmetric form:

$$
\begin{gathered}
\vec{\nabla} \cdot \vec{E}=\left(\mu_{0} c\right) \cdot\left(c \boldsymbol{\rho}_{e}\right) \\
\frac{\partial \vec{E}}{\partial(i c t)}=-\vec{\nabla} \times i c \vec{B}-\left(\mu_{0} c\right) \cdot\left(\overrightarrow{\mathbf{J}}_{e} / i\right) \\
\vec{\nabla} \cdot(i c \vec{B})=\left(i \mu_{0 m} c\right) \cdot\left(c \boldsymbol{\rho}_{m}\right) \\
\frac{\partial(i c \vec{B})}{\partial(i c t)}=-\vec{\nabla} \times \vec{E}-\left(i \mu_{0 m} c\right) \cdot\left(\overrightarrow{\mathbf{J}}_{m} / i\right)
\end{gathered}
$$


where the magnetic permeability caused by moving electric charges $\mu_{0}$ or by magnetic monopoles $\mu_{0 m}$ can be symbolized by the complex $\mu=\mu_{0}+i \mu_{0 m}$. The unified complex charge density and current can be set by:

$$
\begin{gathered}
(c \boldsymbol{\rho}) \equiv\left(\mu_{0} c\right)\left(c \boldsymbol{\rho}_{\boldsymbol{e}}\right)+\left(i \mu_{0 m} c\right)\left(c \boldsymbol{\rho}_{\boldsymbol{m}}\right) \\
(\overrightarrow{\mathbf{J}} / i) \equiv\left(\mu_{0} c\right)\left(\overrightarrow{\mathbf{J}}_{\boldsymbol{e}} / i\right)+\left(i \mu_{0 m} c\right)\left(\overrightarrow{\mathbf{J}}_{\boldsymbol{m}} / i\right)
\end{gathered}
$$

The compact equations become:

$$
\begin{gathered}
\vec{\nabla} \cdot \vec{G}=c \rho \\
\left(\frac{\partial}{\partial i c t}+\vec{\nabla} \times\right) \vec{G}=i \vec{J}
\end{gathered}
$$

Moreover, we define the 4-current:

$$
\mathbf{j}=\left(\begin{array}{c}
c \boldsymbol{\rho} \\
i \overrightarrow{\mathbf{J}}
\end{array}\right)=\left(\mu_{0} c\right) \mathbf{j}_{e}+\left(i \mu_{0 m} c\right) \mathbf{j}_{m}=\left(\mu_{0} c\right)\left(\begin{array}{c}
c \boldsymbol{\rho}_{e} \\
i \overrightarrow{\mathbf{J}}_{\boldsymbol{e}}
\end{array}\right)+\left(i \mu_{0 m} c\right)\left(\begin{array}{c}
c \boldsymbol{\rho}_{m} \\
i \overrightarrow{\mathbf{J}}_{m}
\end{array}\right) \text { with } \mathbf{j}_{\boldsymbol{e}}=\left(\begin{array}{c}
c \boldsymbol{\rho}_{\boldsymbol{e}} \\
i \overrightarrow{\mathbf{J}}_{\boldsymbol{e}}
\end{array}\right), \mathbf{j}_{m}=\left(\begin{array}{c}
c \boldsymbol{\rho}_{m} \\
i \overrightarrow{\mathbf{J}}_{m}
\end{array}\right),
$$

and the 4-E/M-operator:

$$
\hat{\mathrm{L}}_{\mathrm{E} / \mathrm{M}} \equiv\left(\begin{array}{c}
\vec{\nabla} \cdot \\
\frac{\partial}{\partial i c t}+\vec{\nabla} \times
\end{array}\right)
$$

that acts on three-dimensional (3D) vectors to produce 4-vectors, i.e., $\left(\hat{\mathrm{L}}_{\mathrm{E} / \mathrm{M}} \vec{a}\right)_{1 \mathrm{D}}=\vec{\nabla} \cdot \vec{a}$ and $\left(\hat{\mathrm{L}}_{\mathrm{E} / \mathrm{M}} \vec{a}\right)_{3 \mathrm{D}}=\partial \vec{a} / \partial i c t+\vec{\nabla} \times \vec{a}$. Then, the compact and symmetric Maxwell Equations (12) can be written as:

$$
\left(\begin{array}{c}
\vec{\nabla} \cdot \\
\frac{\partial}{\partial i c t}+\vec{\nabla} \times
\end{array}\right) \vec{G}=\left(\begin{array}{c}
c \rho \\
i \vec{J}
\end{array}\right) \text { or } \hat{\mathrm{L}}_{\mathrm{E} / \mathrm{M}} \vec{G}=\mathbf{j}
$$

which in the absence of magnetic monopoles become:

$$
\left(\begin{array}{c}
\vec{\nabla} \cdot \\
\frac{\partial}{\partial i c t}+\vec{\nabla} \times
\end{array}\right) \vec{G}=\left(\mu_{0} c\right) \cdot\left(\begin{array}{c}
c \rho_{e} \\
i \overrightarrow{\mathbf{J}}_{e}
\end{array}\right) \text { or } \hat{\mathrm{L}}_{\mathrm{E} / \mathrm{M}} \vec{G}=\left(\mu_{0} c\right) \cdot \mathbf{j}_{e}
$$

The action of the 4 -Laplace operator $\nabla_{4}$ on $\hat{L}_{\mathrm{E} / \mathrm{M}} \vec{G}$ is:

$$
\nabla_{4} \cdot \hat{\mathrm{L}}_{\mathrm{E} / \mathrm{M}}=\left(-\frac{\partial}{\partial i c t}, \vec{\nabla} \cdot\right)\left(\begin{array}{c}
\vec{\nabla} \cdot \\
\frac{\partial}{\partial i c t}+\vec{\nabla} \times
\end{array}\right)=-\frac{\partial}{\partial i c t}(\vec{\nabla} \cdot)+(\vec{\nabla} \cdot) \frac{\partial}{\partial i c t}+\vec{\nabla} \cdot(\vec{\nabla} \times)=0,
$$

leading to the continuity equation:

$$
0=\nabla_{4} \cdot \mathbf{j}=\left(-\frac{\partial}{\partial i c t}, \vec{\nabla} \cdot\right)\left(\begin{array}{c}
c \boldsymbol{\rho} \\
i \overrightarrow{\mathbf{J}}
\end{array}\right)=-\frac{\partial c \boldsymbol{\rho}}{\partial i c t}+\vec{\nabla} \cdot i \overrightarrow{\mathbf{J}}=i\left(\frac{\partial \rho}{\partial t}+\vec{\nabla} \cdot \overrightarrow{\mathbf{J}}\right) \text { or } \frac{\partial \boldsymbol{\rho}}{\partial t}+\vec{\nabla} \cdot \overrightarrow{\mathbf{J}}=0
$$

Given Equation (11), the continuity equation can be decomposed for the electric and magnetic charge, separately:

$$
\frac{\partial \rho_{e}}{\partial t}+\vec{\nabla} \cdot \overrightarrow{\mathbf{J}}_{e}=0 \text { and } \frac{\partial \rho_{m}}{\partial t}+\vec{\nabla} \cdot \overrightarrow{\mathbf{J}}_{m}=0
$$

\section{Applications}

Next, we examine how the symmetric form of Equation (15) can be applied to the wave equations of fields and gauge transformations. We also obtain the respective equations for potentials. 
First, we define the 4-operator $\hat{\mathrm{L}}_{\mathrm{E} / \mathrm{M}}$ that acts on 4-vectors to produce a $3 \mathrm{D}$ vector.

$$
\hat{\mathrm{L}}_{\mathrm{E} / \mathrm{M}} \equiv\left(\vec{\nabla}, \frac{\partial}{\partial i c t}-\vec{\nabla} \times\right), \hat{\tilde{L}}_{\mathrm{E} / \mathrm{M}}\left(\begin{array}{c}
a_{0} \\
\vec{a}
\end{array}\right)=\vec{\nabla} a_{0}+\frac{\partial \vec{a}}{\partial i c t}-\vec{\nabla} \times \vec{a}
$$

\subsection{Identities}

Below, we examine the action of this 4-operator on two specific 4-vectors, as well the action of the 4-E/M-operator $\hat{\mathrm{L}}_{\mathrm{E} / \mathrm{M}}$ on $\hat{\mathrm{L}}_{\mathrm{E} / \mathrm{M}}$ :

The 4-operator $\hat{\tilde{\mathrm{L}}}_{\mathrm{E} / \mathrm{M}}$ acts on the $\mathrm{E} / \mathrm{M}$ 4-vector operator $\hat{\mathrm{L}}_{\mathrm{E} / \mathrm{M}}$ and produces the wave d'Alembert operator that acts on the $3 \mathrm{D}$ vectors:

$$
\begin{gathered}
\hat{\mathrm{L}}_{\mathrm{E} / \mathrm{M}} \cdot \hat{\mathrm{L}}_{\mathrm{E} / \mathrm{M}}=\left(\vec{\nabla}, \frac{\partial}{\partial i c t}-\vec{\nabla} \times\right)\left(\begin{array}{c}
\vec{\nabla} \cdot \\
\frac{\partial}{\partial i c t}+ \\
+\vec{\nabla} \times
\end{array}\right)=\vec{\nabla}(\vec{\nabla} \cdot)+\frac{\partial^{2}}{\partial(i c t)^{2}}+\frac{\partial}{\partial i c t}(\vec{\nabla} \times)-\vec{\nabla} \times \frac{\partial}{\partial i c t}-\vec{\nabla} \times(\vec{\nabla} \times) \\
=\nabla^{2}+\frac{\partial^{2}}{\partial(i c t)^{2}} \equiv \square^{2}
\end{gathered}
$$

(because $\left.\vec{\nabla} \times(\vec{\nabla} \times)=\vec{\nabla}(\vec{\nabla} \cdot)-\nabla^{2}\right)$.

- The 4-operator $\hat{\tilde{L}}_{\mathrm{E} / \mathrm{M}}$ acts on the gauge transformation 4-vector and vanishes:

$$
\left(\vec{\nabla}, \frac{\partial}{\partial i c t}-\vec{\nabla} \times\right)\left(\begin{array}{c}
-\frac{\partial}{\partial i c t} \\
\vec{\nabla}
\end{array}\right)=-\vec{\nabla} \frac{\partial}{\partial i c t}+\frac{\partial}{\partial i c t} \vec{\nabla}-(\vec{\nabla} \times) \vec{\nabla}=0 .
$$

- The action of the 4-E/M-operator $\hat{\mathrm{L}}_{\mathrm{E} / \mathrm{M}}$ on $\hat{\mathrm{L}}_{\mathrm{E} / \mathrm{M}}$

$$
\hat{\mathrm{L}}_{\mathrm{E} / \mathrm{M}} \cdot \hat{\mathrm{L}}_{\mathrm{E} / \mathrm{M}}=\left(\begin{array}{c}
\vec{\nabla} \cdot \\
\frac{\partial}{\partial i c t}+\vec{\nabla} \times
\end{array}\right)\left(\vec{\nabla}, \frac{\partial}{\partial i c t}-\vec{\nabla} \times\right)=\left(\begin{array}{cc}
\nabla^{2} & \frac{\partial}{\partial i c t} \vec{\nabla} . \\
\frac{\partial}{\partial i c t} \vec{\nabla} & \frac{\partial^{2}}{\partial(i c t)^{2}}-\vec{\nabla} \times(\vec{\nabla} \times)
\end{array}\right) .
$$

\subsection{Wave Equations}

The first identity, in Equation (21), can be applied to producing the field wave equations:

$$
\square^{2} \vec{G}=-\hat{\mathrm{L}}_{\mathrm{E} / \mathrm{M}}\left(\begin{array}{c}
c \rho \\
i \overrightarrow{\mathbf{J}}
\end{array}\right),
$$

expanded as follows:

$$
\square^{2} \vec{G}=\left(\vec{\nabla},-\frac{\partial}{\partial i c t}+\vec{\nabla} \times\right)\left(\begin{array}{c}
c \boldsymbol{\rho} \\
i \overrightarrow{\mathbf{J}}
\end{array}\right)=\vec{\nabla}(c \boldsymbol{\rho})-\frac{\partial(i \overrightarrow{\mathbf{J}})}{\partial i c t}+\vec{\nabla} \times(i \overrightarrow{\mathbf{J}}),
$$

which can be further decomposed to the specific wave equations of the electric field:

$$
\begin{gathered}
\square^{2} \vec{E}=\left(\mu_{0} c\right) \vec{\nabla}\left(c \boldsymbol{\rho}_{e}\right)-\left(\mu_{0} c\right) \frac{\partial \overrightarrow{\mathbf{J}}_{e}}{\partial c t}-\left(\mu_{0 m} c\right) \vec{\nabla} \times \overrightarrow{\mathbf{J}}_{m}, \text { or } \\
\square^{2} \vec{E}=\frac{1}{\varepsilon_{0}} \vec{\nabla} \rho_{e}-\mu_{0} \frac{\partial \overrightarrow{\mathbf{J}}_{e}}{\partial t}-\mu_{0 m} c \vec{\nabla} \times \overrightarrow{\mathbf{J}}_{m} .
\end{gathered}
$$

and the magnetic field:

$$
\square^{2}(c \vec{B})=\left(\mu_{0 m} c\right) \vec{\nabla}\left(c \boldsymbol{\rho}_{m}\right)-\left(\mu_{0 m} c\right) \frac{\partial \overrightarrow{\mathbf{J}}_{m}}{\partial c t}+\left(\mu_{0} c\right) \vec{\nabla} \times \overrightarrow{\mathbf{J}}_{\boldsymbol{e}} \text {, or }
$$




$$
\square^{2} \vec{B}=\frac{1}{\varepsilon_{0 m}} \vec{\nabla} \rho_{m}-\mu_{0 m} \frac{\partial \overrightarrow{\mathbf{J}}_{m}}{\partial t}+\mu_{0} c \vec{\nabla} \times \overrightarrow{\mathbf{J}}_{e}
$$

\subsection{Gauge Transformations}

The identity in Equation (22) can be applied to producing the gauge transformations. First, the potential representation is written as:

$$
\vec{G}=-\hat{\mathrm{L}}_{\mathrm{E} / \mathrm{M}} \mathrm{A}_{e}=-\left(\vec{\nabla}, \frac{\partial}{\partial i c t}-\vec{\nabla} \times\right) \mathrm{A}_{e}, \mathrm{~A}_{e} \equiv\left(\begin{array}{c}
\Phi_{e} \\
i c \overrightarrow{\mathrm{A}}_{e}
\end{array}\right)
$$

Note that in the presence of magnetic monopoles, the 4-potential becomes:

$$
\begin{gathered}
\vec{G}=-\hat{\mathrm{L}}_{\mathrm{E} / \mathrm{M}} \mathrm{A}=-\left(\vec{\nabla}, \frac{\partial}{\partial i c t}-\vec{\nabla} \times\right) \mathrm{A} \text {, with } \\
\mathrm{A} \equiv \mathrm{A}_{e}+i \mathrm{~A}_{m}=\left(\begin{array}{c}
\Phi \\
i c \overrightarrow{\mathrm{A}}
\end{array}\right)=\left(\begin{array}{c}
\Phi_{e} \\
i c \overrightarrow{\mathrm{A}}_{e}
\end{array}\right)+i\left(\begin{array}{c}
\Phi_{m} \\
i c \overrightarrow{\mathrm{A}}_{m}
\end{array}\right)=\left(\begin{array}{c}
\Phi_{e}+i \Phi_{m} \\
i c \overrightarrow{\mathrm{A}}_{e}-c \overrightarrow{\mathrm{A}}_{m}
\end{array}\right) .
\end{gathered}
$$

which can be decomposed to the respective potential equations of the electric field:

$$
\vec{E}=-\vec{\nabla} \Phi_{e}-\frac{\partial \overrightarrow{\mathrm{A}}_{e}}{\partial t}-c \vec{\nabla} \times \overrightarrow{\mathrm{A}}_{m}
$$

and the magnetic field:

$$
c \vec{B}=-\vec{\nabla} \Phi_{m}-\frac{\partial \overrightarrow{\mathrm{A}}_{m}}{\partial t}+c \vec{\nabla} \times \overrightarrow{\mathrm{A}}_{e}
$$

The gauge transformations can be set as:

$$
\mathrm{A}^{\prime}=\mathrm{A}+\left(\begin{array}{c}
-\frac{\partial}{\partial i c t} \\
\vec{\nabla}
\end{array}\right) \cdot i c \Psi \text {, with } \vec{G}^{\prime}=-\hat{\mathrm{L}}_{\mathrm{E} / \mathrm{M}^{\prime}} \text { and } \vec{G}=-\hat{\mathrm{L}}_{\mathrm{E} / \mathrm{M}} \mathrm{A}
$$

Hence:

$$
\vec{G}^{\prime}=\vec{G}-\left(\vec{\nabla}, \frac{\partial}{\partial i c t}-\vec{\nabla} \times\right)\left(\begin{array}{c}
-\frac{\partial}{\partial i c t} \\
\vec{\nabla}
\end{array}\right) \cdot i c \Psi=\vec{G}
$$

\subsection{Symmetric Complex Maxwell Equations for Potentials}

Finally, using the third identity in Equation (23), we derive the Maxwell equations for the potentials:

$$
\begin{gathered}
\hat{\mathrm{L}}_{\mathrm{E} / \mathrm{M}} \vec{G}=-\left(\hat{\mathrm{L}}_{\mathrm{E} / \mathrm{M}} \cdot \hat{\mathrm{L}}_{\mathrm{E} / \mathrm{M}}\right) \mathrm{A}=\mathbf{J} \text {, i.e., } \\
-\left(\begin{array}{cc}
\nabla^{2} & \frac{\partial}{\partial i c t} \vec{\nabla} \cdot \\
\frac{\partial}{\partial i c t} \vec{\nabla} & \frac{\partial^{2}}{\partial(i c t)^{2}}-\vec{\nabla} \times(\vec{\nabla} \times)
\end{array}\right)\left(\begin{array}{c}
\Phi \\
i \overrightarrow{\vec{A}}
\end{array}\right)=\left(\begin{array}{c}
c \boldsymbol{\rho} \\
i \overrightarrow{\mathbf{J}}
\end{array}\right),
\end{gathered}
$$

which can be decomposed to:

$$
\begin{gathered}
-\nabla^{2} \Phi_{e}-\frac{\partial}{\partial t} \vec{\nabla} \overrightarrow{\mathrm{A}}_{e}=\frac{1}{\varepsilon_{0}} \rho_{e} \\
\frac{\partial}{\partial t} \vec{\nabla} \Phi_{e}+\frac{\partial^{2} \overrightarrow{\mathrm{A}}_{e}}{\partial t^{2}}-c^{2} \vec{\nabla} \times\left(\vec{\nabla} \times \overrightarrow{\mathrm{A}}_{e}\right)=\mu_{0} c^{2} \overrightarrow{\mathbf{J}}_{e}
\end{gathered}
$$


and:

$$
\begin{gathered}
-\nabla^{2} \Phi_{m}-\frac{\partial}{\partial t} \vec{\nabla} \overrightarrow{\mathrm{A}}_{m}=\frac{1}{\varepsilon_{0 m}} \boldsymbol{\rho}_{m} \\
\frac{\partial}{\partial t} \vec{\nabla} \Phi_{m}+\frac{\partial^{2} \overrightarrow{\mathrm{A}}_{m}}{\partial t^{2}}+c^{2} \vec{\nabla} \times\left(\vec{\nabla} \times \overrightarrow{\mathrm{A}}_{m}\right)=\mu_{0 m} c^{2} \overrightarrow{\mathbf{J}}_{m}
\end{gathered}
$$

\subsection{Energy-Momentum}

The energy and momentum of the electromagnetic field can be expressed in terms of the vector $\vec{G} \equiv \vec{E}+i c \vec{B}$. Indeed, the energy (density) is given by $\frac{1}{2} \varepsilon_{0} \vec{E}^{2}$ and $\frac{1}{2} \mu_{0}{ }^{-1} \vec{B}^{2}=\frac{1}{2} \varepsilon_{0}(c \vec{B})^{2}$ for the electric and magnetic fields, respectively, that is, $\frac{1}{2} \varepsilon_{0}\left[\vec{E}^{2}+(\overrightarrow{c B})^{2}\right]$, or:

$$
\mathrm{E}=\frac{1}{2} \varepsilon_{0}\left[\vec{E}^{2}+(c \vec{B})^{2}\right]=\frac{1}{2} \varepsilon_{0}\|\vec{G}\|^{2}=\frac{1}{2} \varepsilon_{0}\left(\vec{G} \cdot \vec{G}^{*}\right)
$$

The momentum is proportional to the pointing vector, $\overrightarrow{\mathrm{p}}=c^{-2} \cdot \vec{S}$, and can be written as:

$$
\overrightarrow{\mathrm{p}}=\varepsilon_{0}(\vec{E} \times \vec{B})=\frac{1}{2} \frac{i \varepsilon_{0}}{c}\left(\vec{G} \times \vec{G}^{*}\right)
$$

Hence, the 4-momentum (in Minkowski space) is expressed by:

$$
\left(i \frac{E}{c}, \overrightarrow{\mathrm{p}}\right)=\frac{1}{2} \frac{i \varepsilon_{0}}{c}\left(\vec{G} \cdot \vec{G}^{*}, \vec{G} \times \vec{G}^{*}\right) .
$$

\section{Conclusions}

In this paper, we presented the complex representation of Maxwell equations, indicating the symmetry between electric and magnetic fields, and concluding with a compact form of equations for the fields and their potentials. Using these compact symmetric forms, the wave equations and gauge transformation of the electric and magnetic fields were derived.

The complex symmetric operator formulation presented here can be used as a different way to express the physical content of Maxwell equations. Nevertheless, the developed formulation of Maxwell equations constitutes a much simpler way of unfolding the Maxwell theory rather than previous complex formulations. The simplicity of the presented formulation was shown in the derivations of the basic concepts of (i) continuity equation; (ii) wave equations; (iii) Maxwell equations for potentials; (iv) gauge transformation; and (v) 4-momentum of E/M field, which are briefly summarized below:

By setting,

- Definitions:

$$
\vec{G} \equiv \vec{E}+i c \vec{B}, \mathrm{~A} \equiv\left(\begin{array}{c}
\Phi \\
i c \overrightarrow{\mathrm{A}}
\end{array}\right), \mathbf{j} \equiv\left(\begin{array}{c}
c \rho \\
i \overrightarrow{\mathbf{J}}
\end{array}\right)
$$

- Maxwell equations:

$$
\left(\begin{array}{c}
\vec{\nabla} \cdot \\
\frac{\partial}{\partial i c t}+\vec{\nabla} \times
\end{array}\right) \vec{G}=\mathbf{j}
$$

- Potential representation:

$$
\vec{G}=-\left(\vec{\nabla}, \frac{\partial}{\partial i c t}-\vec{\nabla} \times\right) \text { A. }
$$

we obtain the derivations: 
(i) Continuity equation: Action of $\left(-\frac{\partial}{\partial i c t}, \vec{\nabla} \cdot\right)$ on Equation (42):

$$
\left(-\frac{\partial}{\partial i c t}, \vec{\nabla} \cdot\right) \mathbf{j}=0
$$

(ii) Wave equations: Action of $\left(\vec{\nabla}, \frac{\partial}{\partial i c t}-\vec{\nabla} \times\right)$ on Equation (42):

$$
\square^{2} \vec{G}=\left(\vec{\nabla},-\frac{\partial}{\partial i c t}+\vec{\nabla} \times\right) \cdot \mathbf{j}
$$

(iii) Maxwell equations for potentials: Action of $\left(\begin{array}{c}\vec{\nabla} \cdot \\ \frac{\partial}{\partial i c t}+\vec{\nabla} \times\end{array}\right)$ on $\left(\vec{\nabla}, \frac{\partial}{\partial i c t}-\vec{\nabla} \times\right)$ in Equation (43):

$$
-\left(\begin{array}{cc}
\nabla^{2} & \frac{\partial}{\partial i c t} \vec{\nabla} \\
\frac{\partial}{\partial i c t} \vec{\nabla} & \frac{\partial^{2}}{\partial(i c t)^{2}}-\vec{\nabla} \times(\vec{\nabla} \times)
\end{array}\right) \mathrm{A}=\left(\begin{array}{c}
\Phi \\
i c \overrightarrow{\mathrm{A}}
\end{array}\right)=\left(\begin{array}{c}
c \boldsymbol{\rho} \\
i \overrightarrow{\mathbf{J}}
\end{array}\right) .
$$

(iv) Gauge transformation: Action of $\left(\vec{\nabla}, \frac{\partial}{\partial i c t}-\vec{\nabla} \times\right)$ in Equation (43) on $\left(\begin{array}{c}-\frac{\partial}{\partial i c t} \\ \vec{\nabla}\end{array}\right)$ :

$$
\mathrm{A}^{\prime}=\mathrm{A}+\left(\begin{array}{c}
-\frac{\partial}{\partial i c t} \\
\vec{\nabla}
\end{array}\right) \cdot i c \Psi, \vec{G}^{\prime}=\vec{G} .
$$

(v) 4-momentum (energy and momentum) of electromagnetic field:

$$
\left(i \frac{E}{c}, \overrightarrow{\mathrm{p}}\right)=\frac{1}{2} \frac{i \varepsilon_{0}}{c}\left(\vec{G} \cdot \vec{G}^{*}, \vec{G} \times \vec{G}^{*}\right)
$$

\section{What's Next}

The presented analysis can trigger theoretical developments and applications that differ from the standard Maxwell equations. For example, it will be very exciting to extend the presented analysis within the context of (i) bicomplex numbers and tessarine algebra, that is a four-dimensional vector space over the reals, two-dimensional over the complex numbers [22]; and (ii) $L^{p}$-spaces [23,24] in nonlinear Maxwell equations [25].

In particular, the linearization of general relativity (weak field limit approximation) [26] makes two fields, the gravitoelectric (that is simply the conventional gravity), and the gravitomagnetic (caused by twist of spacetime, e.g., spinning massive objects) [27] that appears in a frame of reference different from that of a freely moving inertial body.

$$
\begin{array}{cc}
\vec{\nabla} \cdot \vec{E}_{g}=-\frac{\rho_{g}}{\varepsilon_{0 g}} & \vec{\nabla} \cdot \vec{E}=\frac{\rho_{e}}{\varepsilon_{0}} \\
\mu_{0 g} \varepsilon_{0 g} \frac{\partial \vec{E}_{g}}{\partial t}=\vec{\nabla} \times \vec{B}_{g}+\mu_{0 g} \overrightarrow{\mathbf{J}}_{g} & \mu_{0} \varepsilon_{0} \frac{\partial \vec{E}}{\partial t}=\vec{\nabla} \times \vec{B}-\mu_{0} \overrightarrow{\mathbf{J}}_{e} \\
\vec{\nabla} \cdot \vec{B}_{g}=0 & \vec{\nabla} \cdot \vec{B}=0 \\
\frac{\partial \vec{B}_{g}}{\partial t}=-\vec{\nabla} \times \vec{E}_{g} & \frac{\partial \vec{B}}{\partial t}=-\vec{\nabla} \times \vec{E}
\end{array}
$$


where $E_{g}$ is the static gravitational or gravitoelectric field and $B_{g}$ is the gravitomagnetic field; and sources $\rho_{g}$ and $J_{g}$ are the mass density and current density, respectively. The involved constants are defined by:

$$
1 /\left(4 \pi \varepsilon_{0 \mathrm{~g}}\right) \equiv G \text { and } \mu_{0 \mathrm{~g}}=1 /\left(c^{2} \varepsilon_{0 \mathrm{~g}}\right) \equiv 4 \pi G / c^{2},
$$

where $G$ is the conventional gravitational constant; the quasi-particle involved in the gravitational Maxwell equations - the graviton-is characterized by the speed of light at vacuum (similar to a photon). The presented formalism can be applied to both sets of Maxwell equations:

$$
\left(\begin{array}{c}
\vec{\nabla} \cdot \\
\frac{\partial}{\partial i c t}+\vec{\nabla} \times
\end{array}\right) \vec{G}_{g}=\left(\begin{array}{c}
c \boldsymbol{\rho}_{g} \\
i \overrightarrow{\mathbf{J}}_{g}
\end{array}\right),\left(\begin{array}{c}
\vec{\nabla} \cdot \\
\frac{\partial}{\partial i c t}+\vec{\nabla} \times
\end{array}\right) \vec{G}=\left(\begin{array}{c}
c \rho_{e} \\
i \overrightarrow{\mathbf{J}}_{e}
\end{array}\right)
$$

with:

$$
\vec{G}_{g} \equiv \vec{E}_{g}+i c \vec{B}_{g}, \vec{G} \equiv \vec{E}+i c \vec{B}
$$

The algebra of bicomplex numbers can be used to unify the two sets of Maxwell equations. Lastly, it will be interesting to investigate whether gravitons can be involved in large-scale quantization constants (e.g., see the work of Carneiro [28] and Livadiotis \& McComas [29]).

In the example that uses $L^{p}$-norms, we can generalize Equation (15) to:

$$
\left(\begin{array}{c}
\vec{\nabla}_{p} . \\
\frac{\partial}{\partial i c t}+\vec{\nabla}_{p} \times
\end{array}\right) \vec{G}=\left(\begin{array}{c}
c \rho \\
i \vec{J}
\end{array}\right)
$$

by using a well-defined $L^{p}$-normed divergence and curl [30]:

$$
\vec{\nabla} \cdot \vec{u} \rightarrow \vec{\nabla}_{p} \cdot \vec{u} \equiv|\vec{\nabla} \cdot \vec{u}|^{p-2}(\vec{\nabla} \cdot \vec{u}), \vec{\nabla} \times \vec{u} \rightarrow \vec{\nabla}_{p} \times \vec{u} \equiv|\vec{\nabla} \times \vec{u}|^{p-2}(\vec{\nabla} \times \vec{u}) .
$$

Then, we may investigate the generated Maxwell equations.

Acknowledgments: The work was supported in part by the project NNX17AB74G of NASA's HGI Program.

Conflicts of Interest: The author declares no conflict of interest.

\section{References}

1. Bialynicki-Birula, I. Photon Wave Function. Prog. Opt. 1996, 36, 245-294.

2. Aste, A. Complex representation theory of the electromagnetic field. J. Geom. Symmetry Phys. 2012, 28, 47-58.

3. Kulyabov, D.S.; Korolkova, A.V.; Sevastianov, L.A. Spinor representation of Maxwell's equations. J. Phys. Conf. Ser. 2017, 788, 012025. [CrossRef]

4. Fushchich, W.I.; Nikitin, A.G. Symmetries of Maxwell's Equations, 2nd ed.; D. Reidel Publishing Company: Dordrecht, The Netherlands; Boston, MA, USA; Lancaster, Lancashire; Tokyo, Japan, 1987.

5. Red'kov, V.M.; Tokarevskaya, N.G.; Spix, G.J. Majorana-Oppenheimer Approach to Maxwell Electrodynamics. Part I. Minkowski Space. Adv. Appl. Clifford Algebras 2012, 22, 1129-1149. [CrossRef]

6. Varlamov, V.V. Maxwell field on the Poincaré group. Int. J. Mod. Phys. A 2005, 20, 4095. [CrossRef]

7. Helmstetter, J.; Micali, A. About the Structure of Meson Algebras. Adv. Appl. Clifford Algebras 2010, 20, 617-629. [CrossRef]

8. Khan, S.A. An Exact Matrix Representation of Maxwell's Equations. Phys. Scr. 2005, 71, 440. [CrossRef]

9. Laporte, O.; Uhlenbeck, G.E. Application of Spinor Analysis to the Maxwell and Dirac Equations. Phys. Rev. 1931, 37, 1380. [CrossRef]

10. Torres-Silva, H. Chiral Maxwell's Equations as Two Spinor System: Dirac and Majorana Neutrino. J. Electromag. Anal. Appl. 2013, 5, 264-270. [CrossRef]

11. Gsponer, A. On the "Equivalence" of the Maxwell and Dirac Equations. Int. J. Theor. Phys. 2002, 41, 689-694. [CrossRef] 
12. Spichak, S. Invariance of Maxwell's Equations under Nonlinear Representations of Poincaré Algebra. Proc. Inst. Math. NAS Ukraine 2004, 50, 961-964.

13. Epstein, C.L.; Greengard, L. Debye Sources and the Numerical Solution of the Time Harmonic Maxwell Equations. arXiv 2008, arXiv:0808.3369v2

14. Epstein, C.L.; Greengard, L.; O’Neil, M. Debye Sources and the Numerical Solution of the Time Harmonic Maxwell Equations II. arXiv 2011, arXiv:1105.3217v1 [CrossRef]

15. Gindikin, S.G.; Khenkin, G.M.; Steklov, V.A. Complex integral geometry and Penrose's representation of the solutions of Maxwell's equations. Teoreticheskaya i Matematicheskaya Fizika 1980, 43, 32-38. [CrossRef]

16. Penrose, R.; Rindler, W. Spinors and Space-Time: Volume 1, Two-Spinor Calculus and Relativistic Fields; Cambridge University Press: Cambridge, UK, 1987.

17. Stickler, D.C. Integral representation for Maxwell's equations with arbitrary time dependence. Proc. Inst. Electr. Eng. 1967, 114, 169-171. [CrossRef]

18. Hailer, K. Maxwell's equations in the multipolar representation. Phys. Rev. A 1982, 26, 1796-1797. [CrossRef]

19. Bogolubov, N.N., Jr.; Prykarpatski, A.K.; Blackmore, D. Maxwell-Lorentz Electrodynamics Revisited via the Lagrangian Formalism and Feynman Proper Time Paradigm. Mathematics 2015, 3, 190-257. [CrossRef]

20. Rajantie, A. Magnetic monopoles in field theory and cosmology. Philos. Trans. R. Soc. B 2012, 370, 5705-5717. [CrossRef] [PubMed]

21. Minkowski, H. Die Grundgleichungen für die elektromagnetischen Vorgänge in bewegten Körpern (Translation: The Fundamental Equations for Electromagnetic Processes in Moving Bodies); Nachrichten von der Gesellschaft der Wissenschaften zu Göttingen, Mathematisch-Physikalische Klasse; DigiZeitschriften: Göttingen, Germany, 1907-1908; pp. 53-111.

22. Davenport, C.M. An Extension of the Complex Calculus to Four Real Dimensions, with an Application to Special Relativity (M.S.); University of Tennessee: Knoxville, TN, USA, 1978.

23. Livadiotis, G. Approach to general methods for fitting and their sensitivity. Physica A 2007, 375, 518-536. [CrossRef]

24. Livadiotis, G. Chi- $p$ distribution: Characterization of the goodness of the fitting using $L^{p}$ norms. J. Stat. Distrib. Appl. 2014, 1, 4. [CrossRef]

25. Wan, A.T.S.; Laforest, M. Nonlinear Maxwell Equations in Inhomogeneous Media. arXiv 2016, arXiv:1605.06532.

26. Hobson, M.P.; Efstathiou, G.P.; Lasenby, A.N. General Relativity: An Introduction for Physicists; Cambridge University Press: Cambridge, UK, 2006; pp. 490-491.

27. Ryder, L.H. Introduction to General Relativity; Cambridge University Press: Cambridge, UK, 2009; pp. $200-207$.

28. Carneiro, S. The large numbers hypothesis and quantum mechanics. Found. Phys. Lett. 1998, 11, 95. [CrossRef]

29. Livadiotis, G.; McComas, D.J. Evidence of large scale phase space quantization in plasmas. Entropy 2013, 15, 1118-1132. [CrossRef]

30. Wan, A.T.S.; Laforest, M. A posteriori error estimation for the p-curl problem. arXiv 2016, arXiv:1605.06532.

(C) 2018 by the author. Licensee MDPI, Basel, Switzerland. This article is an open access article distributed under the terms and conditions of the Creative Commons Attribution (CC BY) license (http://creativecommons.org/licenses/by/4.0/). 